\title{
Identification of elasto-visco-plastic parameters and characterization of Lüders behavior using Digital Image Correlation and the Virtual Fields Method
}

\author{
Stéphane Avril $^{\text {* }}$, Fabrice Pierron ${ }^{\mathrm{a}}$, Michael A. Sutton ${ }^{\mathrm{b}}$, Junhui Yan ${ }^{\mathrm{b}}$ \\ a: Mechanical Engineering and Manufacturing Research Group \\ Ecole Nationale Supérieure d'Arts et Métiers \\ Rue Saint Dominique, BP508, 51006 Châlons en Champagne, FRANCE \\ b: Department of Mechanical Engineering, \\ University of South Carolina, \\ Columbia, SC 29208, USA
}

\begin{abstract}
In this study, tensile loading experiments are performed on notched steel bars at an average applied strain rate of $1 \mathrm{~s}^{-1}$. Displacement fields are measured across the specimen by coupling digital image correlation (DIC) with imaging using high speed CCD cameras (4796 fps). Results from the experiments indicate the presence of local strain rates ranging from 0.1 to $10 \mathrm{~s}^{-1}$ in the notched specimens. The heterogeneity of the strain rate fields provides suitable conditions for determining simultaneously all the elasto-visco-plastic constitutive parameters governing the material behavior. For that, the whole stress fields are reconstructed in the specimen using the fullfield deformation measurements. This reconstruction is repeated with different constitutive parameters until the average stress in the specimen matches the one measured with the load cell response. Perzyna's model is firstly considered for the reconstruction of stresses but it is shown to be unsuited for providing the drop in the average stress that is systematically detected at the onset of plasticity by the load cell. This drop is attributed to the sudden occurrence of plasticity in the material due to Lüders effect. A modified model for elasto-visco-plasticity taking account of Lüders behavior in the material is considered afterwards. It yields a better agreement between the reconstructed stresses and the load cell response, and a more accurate identification of the parameters driving the visco-plastic model. Eventually, it is shown how to use DIC measurements for replacing the load cell measurements when the transient effects in the test reach the resonance frequency of the load cell.
\end{abstract}

Keywords. Viscoplasticity; Digital Image Correlation; Inverse problem; Lüders behavior; Virtual Fields Method; strain rate.

\footnotetext{
* Corresponding author (Dr. Stéphane Avril is now Assistant Professor at Ecole Nationale Supérieure des Mines, SaintEtienne, France. Email address: avril@emse.fr)
} 


\section{Introduction}

Stresses due to inelastic deformation of materials are generally sensitive to strain rate; it is well known that the yield stress of metals increases with strain rate. Knowledge of strain rate sensitivity is necessary for accurate simulation in such situations (e.g., plastic forming, cutting, crashworthiness). Appropriate visco-plastic constitutive models describing the mechanical behavior of materials sensitive to strain rates are employed for achieving these simulations [1].

Since experiments are needed to determine the constitutive parameters of these models, simple mechanical tests (i.e., tension and/or compression of prismatic specimens; torsion of thin tubes) may be carried out. For experiments performed under quasi static conditions, the specimens generally have relatively uniform stress and strain distribution in the gage area of the specimen. By increasing the velocity of the applied displacement/loading, resulting in strain rate increases within the specimen, material parameters governing the visco-plastic behavior can be identified. For example, by plotting the different values of yield stress obtained at different strain rates, an appropriate yield stress versus strain rate model can be defined.

Though simple in concept, this experimental approach has two main drawbacks. First it requires a large number of experiments performed at different strain rates. Second, the assumption of uniform strain and stress distribution is only satisfied under quasi-static conditions; for strain rates greater than $1 \mathrm{~s}^{-1}$, transient strain localization effects cannot be avoided in the specimen [2]. Thus it is not possible to ensure that the strain rate and the stress are constant in the gage length of the specimen. Localization effects can even occur at low strain rates in presence of Lüders effects [3] or PortevinLeChatelier effects [4].

It will be shown in this study that localization effects can be used as an asset if the spatially and temporally varying displacement fields are measured at appropriate time intervals throughout the specimen. Indeed, heterogeneity of strain rate implies that different strain rates occur at different positions in the same specimen, which implies that the measured displacement fields may provide sufficient information for identifying the constitutive parameters governing the elasto-visco-plastic behavior of the material over a certain range of strain rates. 
The measurement of displacement fields, even at moderately high strain rates, is feasible today with modern high speed camera systems [2]. The widespread use of digital image correlation [4,5], denoted DIC, and its coupling with the technology of high-speed cameras, can provide the desired displacement fields. However, the data processing towards the final aim of identifying material constitutive parameters remains an issue. The solution of the inverse problem may be found by minimizing an objective function which compares experimental and simulated data with one another. In this context, uniqueness and stability of the inverse solution affect in particular the identification process. Moreover, simulated data can only be obtained by solving the associated non-linear boundary and initial problem, which is somehow tedious.

Pioneers in the identification of elasto-plastic behavior from full-field measurements were Meuwissen and his coworkers [6]. They suggested performing experiments leading to non uniform stress states, with the idea of retrieving more parameters from a single, well-characterized experiment. The parameters were retrieved by calibrating a finite element (FE) model against the measured displacement fields. This approach is very powerful as the number of parameters identified in a single experiment can be much larger than in classical tensile or torsion loading experiments. Kajberg and his coworkers recently extended this approach to elasto-visco-plastic behavior [2, 7]. However, one of the main drawbacks is that FE computation is time consuming; estimating a few parameters from experimental data can easily take more than 20 hours in some cases [8].

A much more computationally efficient approach for identifying elasto-plastic constitutive parameters from full-field measurements, which does not require any FE computations, has been suggested recently. Originally developed for the identification of elastic properties [9,10,11], the virtual fields method (VFM) has been validated for the identification of elasto-plastic constitutive parameters on simulated data by Grédiac and Pierron [12] and then on experimental data by Pannier et al. [13,14] and Avril et al. [15]. The principle of the VFM for estimating elasto-plastic material parameters is the following: the measured displacement fields are used to quantify the stress components across the specimen. Since the resulting stresses depend upon parameters in the constitutive model via the stress-strain relationships, by requiring the stresses to satisfy equilibrium constraints (via the principle of virtual work), the input material parameters are updated until the equilibrium is satisfied. 
This study is an extension of the VFM to elasto-visco-plasticity. A tensile loading experiment on a double-notched specimen has been specifically designed to give rise to heterogeneous stresses and strain rates during a single test. The investigated material is mild steel (very sensitive to strain rate). In the following sections, the experiments are described. The principle for identifying the elastovisco-plastic constitutive parameters and for characterizing a model of Lüders effect is presented and validated with the experimental data. Eventually, it is shown how to use DIC measurements for replacing the load cell measurements when the transient effects in the test reach the resonance frequency of the load cell.

\section{Standard tensile characterization of elasto-visco-plasticity}

The material used in the experiments is mild steel with $99.5 \%$ pure iron. It is supplied as 2 mm thick rolled sheets from which specimens are cut. Therefore, the material is used in its hardened state and no annealing is considered during this study. A chemical characterization revealed less than $0.02 \%$ carbon in the material. Metallography showed a uniform grain size of about $20 \mu \mathrm{m}$ in average [14]. The grains were mostly composed of ferrite, though grain boundary cementite was locally observed. In order to achieve a standard uniaxial tensile characterization of this material, 18 specimens were machined into a dog-bone shape, with straight edges over a gage length of $60 \mathrm{~mm}$. All of the specimens were cut in the rolling direction of one single rolled sheet so as to avoid a variation of mechanical properties due to the anisotropy induced by rolling. Data for a tensile loading experiment performed at a strain rate of $2 \times 10^{-4} \mathrm{~s}^{-1}$ indicates that the material properties in the transverse and rolling directions are similar, suggesting isotropy in material response is a reasonable approximation [14].

In order to provide reference values for the elasto-visco-plastic constitutive parameters, the material was characterized at different strain rates $\left(2 \times 10^{-4} \mathrm{~s}^{-1}, 4 \times 10^{-2} \mathrm{~s}^{-1}, 1.05 \times 10^{-1} \mathrm{~s}^{-1}, 2.5 \times 10^{-1} \mathrm{~s}^{-1}\right.$,

$4.2 \times 10^{-1} \mathrm{~s}^{-1}$ and $1.05 \mathrm{~s}^{-1}$ ) by standard tensile uniaxial loading experiments on coupons, using 3 samples for each strain rate.

The stress-strain curves obtained from the standard tensile loading experiments are linear before yielding. Young's modulus $E=199 \mathrm{GPa}$ was deduced from the slope of the curves and was independent of strain rate. Poisson's ratio $v=0.28$ was assumed independent of strain rate within the 
range of strain rates considered in this study. It was characterized only at $2 \times 10^{-4} \mathrm{~s}^{-1}$ using strain gage rosette measurements.

Let us define the Von Mises effective stress, denoted $\underline{\sigma}$, such as:

$$
\underline{\sigma}=\sqrt{\frac{3}{2} \sum_{i, j} s_{i j} s_{i j}}
$$

where $s$ is the deviatoric stress tensor with components $s_{i j}$ defined such as $s_{i j}=\sigma_{i j}-\frac{1}{3} \delta_{i j} \sum_{k} \sigma_{k k}$.

The stress-strain curves after yielding are non linear (Fig. 1). Conventional models based on the Von Mises yield function will be used in this study to model the elastic domain evolution of the material [1]. The Von Mises yield function, denoted $f$, may be written:

$$
f(\sigma)=\underline{\sigma}-\sigma_{s}
$$

where $\sigma_{s}$ is the yield stress. The behavior is assumed isotropic and kinematic hardening internal stresses are neglected. The Von Mises yield surface, which defines the elastic domain for a material under multiaxial loading, contains all stress components such as $f(\sigma)=0$.

In order to take isotropic hardening into account, the yield stress $\sigma_{s}$ may be linked to the effective plastic strain, denoted $p$, and defined as:

$$
p(t)=\int_{0}^{t} \sqrt{\frac{2}{3} \dot{\varepsilon}^{p}(t): \dot{\varepsilon}^{p}(t)} d t
$$

where $\dot{\varepsilon}^{p}$ is the plastic strain rate tensor, with components $\dot{\varepsilon}_{i j}^{p}$, and $t$ is the time.

In a standard tensile loading experiment along the $x$ axis, before the occurrence of necking and providing that localization effects are avoided, the loading path is proportional [1]. Thus, the effective plastic strain may be approximated according to:

$$
p(t)=\varepsilon_{y y}(t)-\frac{\sigma_{y y}(t)}{E}=\varepsilon_{y y}(t)-\frac{F(t)}{E S}
$$

where $x$ is the direction of tension and $F$ is the measured resultant load.

Only isotropic hardening is considered in this study. Using the experimental uniaxial tensile loading results for the present material, the relationship between yield stress $\sigma_{s}$ and effective plastic strain can be written:

$$
\sigma_{s}(p)=Y_{0}+H(p)
$$


where $Y_{0}$ is the initial yield stress and $H(p)$ is the hardening function, such as $H(0)=0$. Different functions may be considered for $H(p)$ [1]. Only its initial slope, denoted $E_{t}$, was investigated here. It is defined as:

$$
E_{t}=\frac{d H}{d p}(p=0)
$$

$Y_{0}$ was identified from the stress/strain curves by detecting the stress for which there is a sudden change of slope at the end of elasticity (Fig. 1). $E_{t}$ was identified by fitting linearly in the least squares sense the stress/strain curves after the onset of plasticity within the limit $\varepsilon_{y y}<0.03$.

It can clearly be seen from the curves plotted in Fig. 1 and from the results of Tab. 1 that $E_{t}$ and $Y_{0}$ both depend on the strain rate.

The hardening modulus $E_{t}$ decreases when the strain rate increases (Tab. 1). By looking closely at the curves in Fig. 1, one can notice the existence of a plateau just after the onset of plasticity for strain rates $0.42 \mathrm{~s}^{-1}$ and $1.05 \mathrm{~s}^{-1}$. This plateau almost does not exist for strain rates $0.04 \mathrm{~s}^{-1}$ and $0.1 \mathrm{~s}^{-1}$. This may indicate the development of Lüders behavior for this material when the strain rate becomes large, which is quite common for mild steel [3,16,17]. Lüders behavior exists in annealed mild steel specimens at any strain rate, but also in hardened mild steel specimens (like the ones investigated here) at high strain rates. This phenomenon results from the rapid multiplication of mobile dislocations and the stress-dependence of dislocation velocity at the microstructural scale. It induces the initiation of bands at the shoulder corners of dogbone shaped specimens. After their initiation, the bands move towards the center of the specimen and eventually the strain recovers its uniformity. During the propagation of Lüders band, the stress remains almost constant. Then, hardening is negligible (sometimes even considered negative [1]), which explains why $E_{t}$ is lower for the highest strain rate in Tab. 1. However, for engineering strains larger than 0.05, all the curves in Fig. 1 are parallel, which means that the hardening function $H(p)$ is only affected by the strain rate for small $p$ but is independent of the strain rate for larger $p$ (superior to 0.05). It can be concluded that the effect of strain rate on the hardening of our material disappears when Lüders effect disappears.

Before considering Lüders behavior through appropriate equations (see Section 6), let us focus only on the initial yield stress $Y_{0}$. It is also very sensitive to the strain rate, showing that the investigated material has a visco-plastic behavior. The rate dependent model chosen here for coupling $Y_{0}$ and the strain rate is an additive one suggested by Lemaitre and Chaboche [1]: 


$$
Y_{0}=\sigma_{0}+K_{a} \dot{p}^{\frac{1}{n}}
$$

where $\sigma_{0}$ is the quasi-static initial yield stress (the "drag" stress [3]), $K_{a}$ is the stress rate sensitivity factor and $n$ is the strain rate sensitivity exponent (thus $1 / n$ is the stress rate sensitivity exponent). The values deduced for this model using the data from Tab. 1 are: $\sigma_{0}=140 \mathrm{MPa}, K_{a}=153 \mathrm{MPa} \cdot \mathrm{s}^{-1 / \mathrm{n}}$ and $n=8$.

For simulating numerically the behavior of metals, models giving the plastic strain rate in function of the yield stress are required instead of models such as Eq. (7) that give the yield stress in function of the plastic strain rate. Perzyna's model [2] fulfils this requirement for instance. Perzyna's model is deduced from Eq. (7) by a mere inversion. Accordingly, the equivalent plastic strain rate is deduced from the yield stress using the following equation:

$$
\dot{p}=\gamma\left\langle\frac{Y_{0}}{\sigma_{0}}-1\right\rangle^{n}=\gamma\left\langle\frac{\sigma_{s}-H(p)}{\sigma_{0}}-1\right\rangle^{n}
$$

where $\langle z\rangle=z$ if $z>0,\langle z\rangle=0$ otherwise. Eq. (8) is only satisfied when $f(\sigma)=0$, i.e. when $\underline{\sigma}=\sigma_{s}$ (the yield stress equals the effective stress). Otherwise, the material is in the elastic range $\left(\underline{\sigma}<\sigma_{s}\right)$ and consequently, $\dot{p}=0$.

Parameter $\gamma$, which is the strain rate sensitivity factor and has the dimension of strain rate, is defined such as:

$$
\gamma=\left(\frac{\sigma_{0}}{K_{a}}\right)^{n}
$$

The value deduced for this parameter using the data from Tab. 1 is $\gamma=0.5 . \mathrm{s}^{-1}$.

\section{Novel approach for identifying elasto-visco-plastic constitutive parameters}

\subsection{Experiment and specimen}

A tensile loading experiment was specifically designed to give rise to heterogeneous distributions of stresses and strain rates across a flat thin specimen with notches. Fig. 2 shows the notched bar tensile specimen. The displacement ramp rate is about $61 \mathrm{~mm} / \mathrm{s}$. The data sampling rate for recording the resultant load applied by the MTS tensile machine is $1 \mathrm{kHz}$. Two high speed cameras were used for synchronized recording of images of the front and back surfaces of a nominally planar specimen; images of each surface are required so that data measured on both sides could be compared to detect 
possible parasitic effects of out-of-plane motion on the deformation measurements [13]. The imaging was performed at 4796 frames per second with a full resolution of $800 \times 600$ pixels.

Experiments were performed with two specimens. The resultant load measured during both tests has been plotted in Fig. 3, showing a good repeatability.

The deformation of the specimens during loading was analyzed by the DIC technique with the help of the VIC-2D software [18] using a $21 \times 21$ pixel correlation window and a step size of 5 pixels between subset centers. The measurements have been estimated to have a positional error of about $10^{-2}$ pixel. For filtering purposes, the measured displacement fields were projected on a basis of piecewise linear functions, denoted $f_{k}[11]$. The geometry of the specimen in the measurement area was meshed using triangles with a mesh size of about $4 \mathrm{~mm}$ (Fig. 2). Each function $f_{k}$ equals one at a given node of the mesh and zero at all the other nodes (shape functions). The reconstructed displacement fields can be written:

$$
\begin{aligned}
& U_{x}(x, y)=\sum_{k} a_{k} f_{k}(x, y) \\
& U_{y}(x, y)=\sum_{k} b_{k} f_{k}(x, y)
\end{aligned}
$$

where $a_{k}$ and $b_{k}$ were obtained by regression in the least squares sense. Then, the displacement fields reconstructed with the basis functions were used to deduce the strain fields required for the identification, with an estimated resolution (least detectable strain, characterized from two different images of the undeformed specimen) of $2 \cdot 10^{-5}$ and a spatial resolution (least distance between two uncorrelated strain values derived from the measurements) of $4 \mathrm{~mm}$. The maps of strain component $\varepsilon_{y y}$ are shown in Fig. 4. Localization of strains at the center of the specimen is evident.

Differentiating the strain fields with time provides maps of the strain rate, as for instance $d \varepsilon_{y y} / d t$ in Fig. 5. The maps shown in Fig. 5 were obtained without any further filtering than the reconstruction according to Eq. (10). As the strain resolution is $2 \cdot 10^{-5}$ and the acquisition frequency is 4796 images per second, the strain rate resolution is about $4796 \times 2.10^{-5} \approx 0.1 \mathrm{~s}^{-1}$, with a spatial resolution of $4 \mathrm{~mm}$ again and a time resolution of $0.2 \mathrm{~ms}(=1 / 4796)$. Such a short time resolution makes possible the detection of very short-duration phenomena in the response of the specimen. Interestingly, the strain rate fields are heterogeneous, varying by nearly two decades within the same image, between $0.1 \mathrm{~s}^{-1}$ (strain rate resolution) and $8 \mathrm{~s}^{-1}$, even though the average strain rate is held constant throughout the experiment by prescribing a constant speed to the cross head. Since the same phenomenon was observed for the two tested specimens, the following discussion is provided. 
High values of strain rate are clearly observed in the center of the specimen about $7 \mathrm{~ms}$ after initiating the displacement ramp (see Fig. 5). The plastic strain rate in the central part of the specimen overtakes the local prescribed strain rate that occurred during the elastic stage, inducing a redistribution of strains and also a decrease of the measured resultant load (see Fig. 3). The large strain rates in the center of the specimen are compensated by a quasi-zero strain rate away from the center. Therefore, for the same ramping rate of the tensile machine, much larger strain rates could be reached because only a localized part of the specimen continues to be deformed. The transition from a quasi-uniform (within measurement uncertainty) strain rate distribution during the elastic behavior to a localized strain rate distribution after the onset of plasticity provides conditions for characterizing the visco-plastic behavior in a single experiment. The following sections show how the strain fields can be processed to retrieve the constitutive parameters governing this behavior.

\subsection{The VFM in the elastic range}

The global equilibrium of a solid with neither body nor acceleration forces acting on it can be written as:

$$
-\sum_{i, j} \int_{V} \sigma_{i j} \varepsilon_{i j}^{*} d V+\sum_{i} \int_{\partial V} T_{i} \cdot u_{i}^{*} d S=0
$$

This equation is referred to as the "principle of virtual work". The $\sigma_{i j}$ are the component of the stress tensor across the volume of the solid (denoted $V$ ), the $T_{i}$ are the components of a vector figuring the surface density of the traction applied over the external surface of the solid (denoted $\partial V$ ), the $u_{i}{ }^{*}$ are the components of a vectorial test function which only has to be continuous across the solid. This test function is named "virtual displacement field" and the $\varepsilon_{i j}{ }^{*}$ are the components of the virtual strain tensor derived from it.

Let us assume that the investigated specimen is in a plane stress state. This is relevant due to the thinness of the plate, its constant thickness (denoted $h$ in the following) and to the applied loading (in-plane tension). Thus the stress can be assumed homogeneous across the thickness, along with the strain and the deformation.

Before yielding (the elastic behavior range lasts about $6 \mathrm{~ms}$ ), the material is elastic and isotropic. During this time, the stress components can be expressed as a linear function of the strain components (using the convention of contracted indices $(x x \rightarrow x, y y \rightarrow y, x y \rightarrow s)$ : 


$$
\left(\begin{array}{c}
\sigma_{x} \\
\sigma_{y} \\
\sigma_{s}
\end{array}\right)=\frac{E}{1-v^{2}}\left[\begin{array}{ccc}
1 & v & 0 \\
v & 1 & 0 \\
0 & 0 & \frac{1-v}{2}
\end{array}\right]\left(\begin{array}{c}
\varepsilon_{x} \\
\varepsilon_{y} \\
\varepsilon_{s}
\end{array}\right)
$$

Thanks to the measurement of the displacement fields and their projection on the basis of piecewise linear function shown in Eq. (10), the in-plane strain fields are known across a whole area of interest in the specimen. Writing Eq. (11) over this area, one has:

$$
\frac{E}{1-v^{2}}\left[\int_{S}\left(\varepsilon_{x} \varepsilon_{x}^{*}+\varepsilon_{y} \varepsilon_{y}^{*}+\frac{1}{2} \varepsilon_{S} \varepsilon_{S}^{*}\right) d S+v \int_{S}\left(\varepsilon_{y} \varepsilon_{x}^{*}+\varepsilon_{x} \varepsilon_{y}^{*}-\frac{1}{2} \varepsilon_{S} \varepsilon_{s}^{*}\right) d S\right]=\frac{1}{h} \int_{\partial V} T_{i} \cdot u_{i}^{*} d S
$$

The latter equation is satisfied with any virtual field $u_{i}{ }^{*}[9]$. Poisson's ratio is identified by using a virtual field that figures a lateral shrinking deformation and for which the contribution of the resultant load is cancelled. This virtual field is chosen so as to maximize the identifiability of Poisson's ratio according to the theory developed in [11].

Young's modulus is identified using: $u_{x}{ }^{*}=0$ and $u_{y}{ }^{*}=y$, corresponding to the following virtual strain field: $\varepsilon_{x x}{ }^{*}=0$ and $\varepsilon_{y y}{ }^{*}=1$ and $\varepsilon_{x y}{ }^{*}=0$. Eq. (13) with this virtual field can be written in the form,

$$
\frac{E}{1-v^{2}}\left[\int_{S} \varepsilon_{y}(x, y, t) d S+v \int_{S} \varepsilon_{x}(x, y, t) d S\right]=\frac{F(t) L}{h}
$$

where $t$ is time, $L$ is the length of the area of interest where the measurement is achieved (see Fig. 2) and $F$ is the measured load resultant. The area of the surface of interest where the measurement is achieved is denoted $S$.

Defining $\underline{\varepsilon}_{x}$ and $\underline{\varepsilon}_{y}$ as the average strains over $S$, one gets:

$$
\frac{E}{1-v^{2}}\left[\underline{\varepsilon}_{y}(t)+v \underline{\varepsilon}_{x}(t)\right]=\frac{F(t) L}{S h}
$$

Young's Modulus is identified by plotting $F\left(t_{l}\right) L / S h$ (average stress) versus $\left[\underline{\varepsilon}_{x}\left(t_{l}\right)+v \underline{\varepsilon}_{y}\left(t_{l}\right)\right] /\left(1-v^{2}\right)$ (dimensionless, like strains), for different times $t_{l}$, all along the experiment $(0<k<N)$. The obtained curve is eventually fitted by a linear curve in the elastic domain for estimating $E$.

\section{The VFM in the plastic range}

For plasticity and visco-plasticity, Eq. (11) may be rewritten in a rate dependent form:

$$
-\sum_{i, j} \int_{V}\left[\int_{0}^{t} \dot{\sigma}_{i j} d t\right] \varepsilon_{i j}^{*} d S+\sum_{i} \int_{\partial V} T_{i} \cdot u_{i}^{*} d S=0
$$


where $\dot{\sigma}=d \sigma / d t$ is the stress rate. The stress rate remains constant in linear elasticity, but it generally does not in plasticity and visco-plasticity except when the loading paths are proportional [1]. The test considered in this study does not provide conditions of proportional loading. This is the reason why Eq. (16) must be used instead of Eq. (11) for identifying the visco-plastic parameters. For addressing this identification problem and compute the time integral in Eq. (16), the elasto-visco-plastic constitutive equations may be written as:

$$
\dot{\sigma}=g(\sigma, \dot{\varepsilon}, X)
$$

where $g$ is a given vectorial function of the actual strain rate $\dot{\varepsilon}=d \varepsilon / d t$, of the actual stress $\sigma$ and of the unknown constitutive parameters. The latter are denoted $X$ when designated entirely and $X_{p}$ when designated individually, $1 \leq p \leq P$, where $P$ is the number of unknown constitutive parameters.

The Von Mises yield surface was assumed relevant for the investigated material. Assuming volume conservation in plasticity and full separation between elastic and plastic strains, the following flow rule can be used for deriving function $g$ :

$$
\left\{\begin{array}{l}
\dot{\sigma}_{x} \\
\dot{\sigma}_{y} \\
\dot{\sigma}_{s}
\end{array}\right\}=\frac{E}{1-v^{2}}\left[\begin{array}{ccc}
1 & v & 0 \\
v & 1 & 0 \\
0 & 0 & \frac{1-v}{2}
\end{array}\right]\left\{\begin{array}{l}
\dot{\varepsilon}_{x}-\frac{3}{2} \dot{p} \frac{s_{x x}}{\sigma_{s}} \\
\dot{\varepsilon}_{y}-\frac{3}{2} \dot{p} \frac{s_{y y}}{\sigma_{s}} \\
\dot{\varepsilon}_{s}-3 \dot{p} \frac{s_{x y}}{\sigma_{s}}
\end{array}\right\}
$$

where $s_{x x}, s_{y y}$, and $s_{x y}$ are the in-plane components of the deviatoric stress tensor and $\dot{p}$ is the equivalent plastic strain rate. For modeling the viscoplastic behavior, $\dot{p}$ was derived from Eq. (8) according to Perzyna's model [2]. Therefore, using Eq. (8), the constitutive equations may be written for this particular case:

$$
\left\{\begin{array}{l}
\dot{\sigma}_{x} \\
\dot{\sigma}_{y} \\
\dot{\sigma}_{s}
\end{array}\right\}=\frac{E}{1-v^{2}}\left[\begin{array}{ccc}
1 & v & 0 \\
v & 1 & 0 \\
0 & 0 & \frac{1-v}{2}
\end{array}\right]\left\{\begin{array}{l}
\dot{\varepsilon}_{x}-\frac{3}{2} \gamma\left\langle\frac{\sigma_{s}-H(p)}{\sigma_{0}}-1\right)^{n} \frac{s_{x x}}{\sigma_{s}} \\
\dot{\varepsilon}_{y}-\frac{3}{2} \gamma\left\langle\frac{\sigma_{s}-H(p)}{\sigma_{0}}-1\right)^{n} \frac{s_{y y}}{\sigma_{s}} \\
\dot{\varepsilon}_{s}-3 \gamma\left(\frac{\sigma_{s}-H(p)}{\sigma_{0}}-1\right\rangle^{n} \frac{s_{x y}}{\sigma_{s}}
\end{array}\right\}
$$

Eq. (19) is a first order differential equation where function $\sigma(x, y, t)$ is unknown. It can be recovered by solving this equation numerically with the Euler scheme. Time is discretized, each step corresponding to times for which measurements are achieved. Increment of $\sigma(x, y, t)$ from one step to another are assessed according to: 


$$
\left\{\begin{array}{l}
\sigma_{x}(t+\Delta t) \\
\sigma_{y}(t+\Delta t) \\
\sigma_{s}(t+\Delta t)
\end{array}\right\}=\left\{\begin{array}{l}
\sigma_{x}(t) \\
\sigma_{y}(t) \\
\sigma_{s}(t)
\end{array}\right\}+\frac{E}{1-v^{2}}\left[\begin{array}{ccc}
1 & v & 0 \\
v & 1 & 0 \\
0 & 0 & \frac{1-v}{2}
\end{array}\right]\left\{\begin{array}{l}
\varepsilon_{x}(t+\Delta t)-\varepsilon_{x}(t)-\frac{3}{2} \gamma\left\langle\frac{\sigma_{s}(t)-E_{t} p(t)}{\sigma_{0}}-1\right\rangle^{n} \frac{s_{x x}(t)}{\sigma_{s}(t)} \Delta t \\
\varepsilon_{y}(t+\Delta t)-\varepsilon_{y}(t)-\frac{3}{2} \gamma\left\langle\frac{\sigma_{s}(t)-E_{t} p(t)}{\sigma_{0}}-1\right\rangle^{n} \frac{s_{y y}(t)}{\sigma_{s}(t)} \Delta t \\
\varepsilon_{s}(t+\Delta t)-\varepsilon_{s}(t)-3 \gamma\left\langle\frac{\sigma_{s}(t)-E_{t} p(t)}{\sigma_{0}}-1\right\rangle^{n} \frac{s_{x y}(t)}{\sigma_{s}(t)} \Delta t
\end{array}\right\}
$$

where $\Delta t$ is the time increment between two consecutive measurements. The first order approximation of the hardening function $H(p) \approx E_{t} p$ is assumed relevant. This assumption is justified by the small strains for which Eq. (20) will be applied further. It will be discussed in Section 6.

The initial condition for launching the Euler scheme is that the behavior is elastic, meaning that Eq. (12) stands instead of Eq. (20) at the first iteration. A special attention is paid to the convergence of the Euler scheme. A slight mismatch between the computed stress and the actual yield stress occurs due to the approximations introduced in Eq. (20) by the time discretization and linearization of the stress increment from one step to another. This mismatch is corrected by the method of radial return, as explained by Sutton et al. [19] and as already used in elasto-plasticity by Avril et al. [15]. Therefore, from the available full-field measurements, and from a given set of constitutive parameters $\left(\sigma_{0}, n, \gamma, E_{t}, E\right.$ and $\left.v\right)$, stress fields can be derived across the area of interest of the specimen all along the test using Eq. (20).

Using these stress fields, it is possible to assess the suitability of the parameters $\sigma_{0}, n, \gamma, E_{t}$ input into the algorithm. Parameters $E$ and $v$ have been deduced from the strain fields measured before the onset of plasticity and therefore, one only needs to check the suitability of $\sigma_{0}, n, \gamma, E_{t}$ during the postelastic deformation process. This is achieved simply by applying the principle of virtual work written in Eq. (16), with: $u_{x}{ }^{*}=0$ and $u_{y}{ }^{*}=y$, corresponding to the following virtual strain field: $\varepsilon_{x x}{ }^{*}=0$ and $\varepsilon_{y y}{ }^{*}=1$ and $\varepsilon_{x y}{ }^{*}=0$. Accordingly, the following equation should be verified at each time $t$ during the experiment:

$$
\int_{S} \int_{0}^{t} \dot{\sigma}_{y}\left(\sigma_{0}, n, \gamma, E_{t}, x, y, t\right) d t d S=\frac{P(t) L}{h}
$$

Since experimental noise in the data will introduce errors in Eq (21), the idea is to compute repeatedly the stress fields from Eq. (20) and to update $\sigma_{0}, n, \gamma, E_{t}$ until the minimum of the following cost function is reached: 


$$
F\left(\sigma_{0}, n, \gamma, E_{t}\right)=\sum_{l=1}^{N}\left(\frac{1}{S} \int_{S} \int_{0}^{t_{l}} \dot{\sigma}_{y}\left(\sigma_{0}, n, \gamma, E_{t}, x, y, t\right) d t d S-\frac{P\left(t_{l}\right) L}{S h}\right)^{2}
$$

Eq. (22) gives the quadratic deviation between the average longitudinal stress computed from the measured displacement fields and the average longitudinal stress computed from the measured resultant load, summed up over the duration of the experiment. The evolution of both average stresses has been plotted in Fig. 6 at the minimum of the cost function. This minimum is generally reached in about 50 iterations by using a Nelder-Mead algorithm (about two minutes of time computation using a Pentium M, $1400 \mathrm{MHz}$ ). This represents a dramatic reduction of the computation time compared to the use of finite element model updating approaches that require several hours to obtain similar results [8].

\section{Experimental results and discussion of Perzyna's model}

The material parameters obtained using the approach presented in the previous sections are reported in Tab. 2. The estimated material parameters have been obtained for each specimen by using the two sets of data from separate high speed cameras on each side of the specimen to independently compute the parameters. The results are compared with the reference that was identified using standard tension test on the dogbone specimen.

Regarding the elastic parameters, Young's modulus identified on both sides of the two specimens are the same and in agreement with the reference. Since Young's modulus is the same on both sides, it can be concluded that bending effects and out of plane parasitic motions discussed by Pannier et al. [13] are negligible in these experiments. Regarding Poisson's ratio, they are also in good agreement with the reference. Variations exist between both sides of the same specimen (Tab. 2). It is conjectured that data scattering may be responsible for these variations.

Regarding the visco-plastic parameters, Tab. 2 shows that there is a general agreement with the reference values for $\sigma_{0}$ but deviations from the reference values remain large for parameters such as $\gamma$ and $n$. The hardening modulus has no counter part in the reference experiments because the localized strain rates reached in the notched bar could not be reached in the dog-bone specimen. Nevertheless, it is noted that the values of $E_{t}$ identified in the notched bars are always lower than the ones identified in the dogbone specimens. This trend is in agreement with a reduction of hardening as 
strain rate increases, as shown in Tab. 1. It may indicate an amplification of Lüders effect in the double notched compared to the dogbone specimens. This will be discussed in Section 6 .

Deviations for $\gamma$ and $n$ may be explained by different reasons. The reference experiments cover only a few decades of strain rates, with only six different values. This may be insufficient to accurately estimate the three parameters in Perzyna's model. Experiments carried out on the notched bars with a full-field measurement technique provide richer information, as each triangle defined in the measurement area (Fig. 2) corresponds to an independent measurement. Such a density of data can be used to assess the relevancy of Perzyna's model when used to model the present material behavior. This was achieved by comparing the evolution of two stresses:

1. the average stress deduced from the displacement fields, which is the first term of cost function $F\left(\sigma_{0}, n, \gamma, E_{t}\right)$ in Eq. (22):

$$
\sigma_{A V 1}\left(\sigma_{0}, n, \gamma, E_{t}, t_{l}\right)=\frac{1}{S} \int_{S} \int_{0}^{t_{l}} \dot{\sigma}_{y}\left(\sigma_{0}, n, \gamma, E_{t}, x, y, t\right) d t d S
$$

2. the average stress deduced from the resultant load, which is the second term of cost function $F\left(\sigma_{0}\right.$, $\left.n, \gamma, E_{t}\right)$ in Eq. (22):

$$
\sigma_{A V 2}\left(t_{l}\right)=\frac{P\left(t_{l}\right) L}{S h}
$$

It can be deduced from Fig. $6 \mathrm{a}$ that, even for the parameters $\sigma_{0}, n, \gamma$ and $E_{t}$ that provide the minimum of cost function $F$, poor agreement exists between $\sigma_{A V 1}$ and $\sigma_{A V 2}$; the drop after the maximum and the oscillations of $\sigma_{A V 2}$ could not be reproduced in $\sigma_{A V 1}$ from the displacement fields. More detailed investigations by the authors indicated that no set of values could be found for $\sigma_{0}, n, \gamma, E_{t}$ that would result in $\sigma_{A V I}$ where such oscillations occur. Accordingly, Perzyna's model in Eq. (8) seems unsuited to model the observed behavior.

The unsuitability of Perzyna's model may account for errors obtained for exponent $n$ in Tab. 2. Indeed, the cost function is only sensitive to this parameter at the onset of plasticity, between $6 \mathrm{~ms}$ and $9 \mathrm{~ms}$, when the distribution of strain rates is the widest (varying between $0.1 \mathrm{~s}^{-1}$ and $8 \mathrm{~s}^{-1}$, according to the maps plotted in Fig. 5). As the model is unsuited during this part of the experiment (it cannot provide oscillations for $\sigma_{A V I}$ ), it is unlikely that the exponent $n$ in Tab. 2 can be correctly estimated. For the same reason, values reported in Tab. 2 for $E_{t}$ are probably also affected. 


\section{Results with an improved model taking account of Lüders behavior}

As shown in Fig. 6a, a rough agreement between $\sigma_{A V 1}$ and $\sigma_{A V 2}$ exists, except in the transient part when the drop and then oscillations occur in the load versus time curve (Fig. 3). To account for this particular behavior in the transient part, a model of Lüders behavior has to be introduced in the equations because Lüders effect becomes predominant during this part of the test due to the high strain rate.

Indeed, the zone of localized strain rate in Fig. 5 may actually be viewed as a Lüders band. Lüders bands occur usually in annealed mild steel [16]. Sharp yield points, abrupt yield drops and subsequent plateau are generally noticed in the stress/strain curves of standard uniaxial tensile tests [3]. This can also occur in non-annealed mild steel at high strain rates, as this was observed for our material in the standard uniaxial tensile tests carried out on the dogbone specimens (Fig. 1). This phenomenon results from the rapid multiplication of mobile dislocations (like an avalanche) and the stress-dependence of dislocation velocity. It induces the initiation of bands at the shoulder corners of the standard dogbone specimens. Afterwards, the bands move towards the center of the specimen and eventually the strain recovers its uniformity. It is different in the double-notched specimen used here because, due to the specimen geometry, the Lüders bands are unable to escape from the notched zone [17]. Then, the Lüders bands just disappear when the dislocation multiplication ends.

Lüders effects is actually independent of hardening properties [3, 16]. A hardening function such as $H(p)=E_{t} p$ still provides satisfactory approximations of hardening for our study within the range of strains which are considered. Lüders effect is rather modelled as a variation of the initial yield stress $Y_{0}$ in the literature $[3,16]$. Indeed, in order to model this effect, one has to consider that $\gamma$ in Eq. (13) or (14) depends on the cumulative plastic strain $p[3,16] ; \gamma$ is the product of the number of dislocations by the elementary velocity of each dislocation (norm of the Burgers vector [1]). If the number of mobile dislocations changes, $\gamma$ cannot be assumed as constant. A general model suited for Lüders behavior is the Yoshida's model $[3,16]$. It may be written:

$$
\gamma=\gamma_{\infty}+\left(\gamma_{0}-\gamma_{\infty}\right) e^{-\lambda p}
$$

Therefore, let us consider the following model as an extension of Perzyna's model to loading configuration where the plastic strain increment may vary very fast:

$$
\dot{p}(t)=\left[\gamma_{\infty}+\left(\gamma_{0}-\gamma_{\infty}\right) e^{-\lambda p(t)}\right]\left\langle\frac{\sigma_{s}(t)-E_{t} p(t)}{\sigma_{0}}-1\right\rangle^{n}
$$


where $\lambda, \gamma_{0}$ and $\gamma_{\infty}$ are three constants to determine. If $\lambda$ is close to zero, the material is almost not affected by the Lüders effect and Perzyna's usual model is recovered, with $\gamma=\gamma_{0}$.

Accordingly, increments of $\sigma(x, y, t)$ from one step to another are now assessed according to:

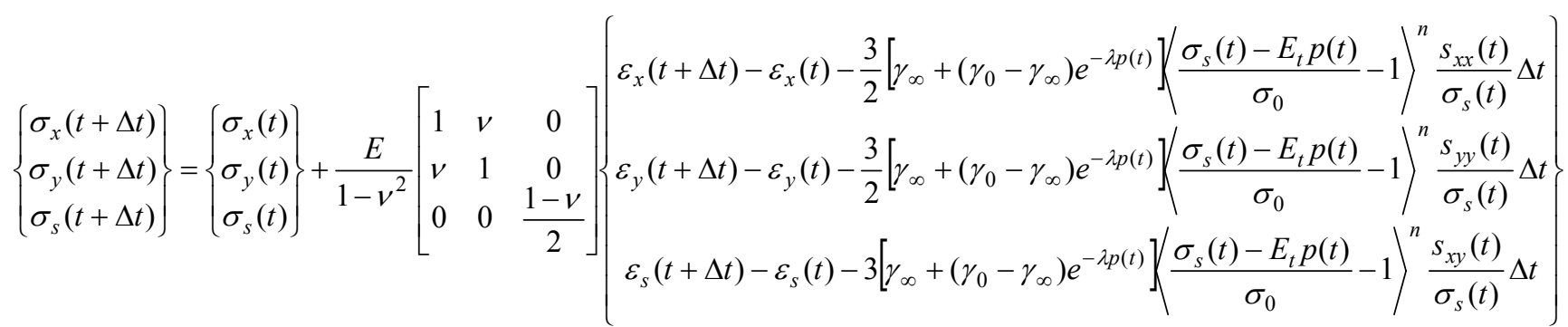

Using Eq. (27) instead of Eq. (20) for deriving $\sigma_{A V I}$ from the measured displacements, one obtains improved agreement with $\sigma_{A V 2}$ in the transient stage. For example, the curve deduced from displacement fields in Fig. 6b was obtained with parameters:

$\sigma_{0}=134 \mathrm{MPa}, \gamma_{0}=0.007 \mathrm{~s}^{-1}, \gamma_{\infty}=0.2 \mathrm{~s}^{-1}, E_{t}=1.6 \mathrm{GPa}, n=5.4$ and $\lambda=26$.

These parameters are the ones for which the best agreement between both curves in Fig. $6 \mathrm{~b}$ is reached. Parameters identified for the other side of the specimen and for the other test are reported in Tab. 3. Discrepancies between parameters obtained on the same specimen but using the data from the two different cameras located on each side of the specimen gives an idea of the sensitivity to measurement errors of the approach. Indeed, similar parameters should be identified on both sides of the same specimen. Variations of parameters $E_{t}, \sigma_{0}$ and $n$ from one side to another are less than $10 \%$. These parameters are more stable than the parameters of Yoshida's model $\gamma_{0}, \gamma_{\infty}$, and $\lambda$, which are identified with variations reaching more than $50 \%$ from one side to the other in the same specimen. This indicates that the test is not really appropriate for characterizing the Lüders effect, which is more like a marginal effect here.

The initial purpose of this study was not to identify parameters governing a model of Lüders behavior. The initial purpose was to identify the visco-plastic parameters of Perzyna's model and the Lüders effect in the model was only considered to improve this identification. This improvement has been reached as the identified values of $\sigma_{0}, \gamma_{\infty}$, and $n$ reported in Tab. 3 are in better agreement with the reference values compared to the values reported in Tab. 2. This can be checked in Fig. 7 where 
three curves representing the variation of $Y_{0}$, according to Eq. (7) and (9), have been plotted in function of the strain rate (between $10^{-4} \mathrm{~s}^{-1}$ and $100 \mathrm{~s}^{-1}$ ):

- the curve using the parameters derived from the standard tests, i.e. the one that fits at best the data reported in Tab. 1,

- the curve using the average values of $\sigma_{0}, \gamma$, and $n$ reported in Tab. 2, derived from the experiment on the double-notched specimens using the original Perzyna's model,

- the curve using the average values of $\sigma_{0}, \gamma_{\infty}$, and $n$ reported in Tab. 3 , derived from the experiment on the double-notched specimens using the modified Perzyna's model.

A good agreement exists between the curve derived from the standard tests and the curve derived from the parameters reported in Tab. 3. However, the curve derived from the parameters reported in Tab. 2, i.e. identified with the VFM using the original Perzyna's model, is, as expected, largely inaccurate. This proves that the consideration of the modified form of Perzyna's model was required for a successful identification of the visco-plastic behavior using the double-notched specimens.

Moreover, the hardening modulus $E_{t}$ reported in Tab. 3 is less scattered than in Tab. 2. $E_{t}$ is now independent of the strain rate because the effects of strain rates on hardening are modeled through Lüders behavior. This is why the hardening modulus value is now higher than the values reported in Tab. 2. Reference values for $\gamma_{0}, E_{t}$, and $\lambda$ in the Tab. 3 are not reported because Lüders effect in the standard uniaxial tensile tests carried out on the dogbone specimens was not pronounced enough to identify these parameters. The reported reference value of $\gamma_{\infty}$ is the value of $\gamma$ previously identified from Eq. (9).

\section{Addressing resonance issues in the load cell response using DIC measurements}

Even though the modified Perzyna's model provides promising parameter values and a nice drop of $\sigma_{A V I}$ after the onset of plasticity in Fig. 6b, discrepancies remain: there is no oscillation of $\sigma_{A V I}$ after the drop whereas oscillations recorded by the load cell after the onset of plasticity still appear in the curve of $\sigma_{A V 2}$. Actually, the oscillations recorded by the load cell are probably induced by vibrations in the tensile machine, affecting only the load cell data (far-field stress) but not the stresses across the field area. Indeed, a large energy release results from the occurrence of the Lüders band at the onset of plasticity. Significant accelerations (computed from the displacement fields by a double differentiation along the time) can be observed in the measurement area at this time (at $7 \mathrm{~ms}$ in Fig. 8). The bottom of the specimen has an acceleration of more than $2.5 \mathrm{~g}\left(25 \mathrm{~m} / \mathrm{s}^{2}\right)$ and the top 
accelerates in the backward direction at about $-1.5 \mathrm{~g}\left(-15 \mathrm{~m} / \mathrm{s}^{2}\right)$. Vibrations are induced by this sudden acceleration and this results in a reverse acceleration field at $9 \mathrm{~ms}$ : the bottom now accelerates backward and the top forward, as if a shock wave was coming back after being reflected at the cross head of the tensile machine. These vibrations are amplified at the load cell due to the mass of the cross heads and other machine components. Indeed, if a mass of $40 \mathrm{~kg}$ was accelerated at $2.5 \mathrm{~g}$, the load would be amplified by $1000 \mathrm{~N}$, and thus the average stress deduced from the resultant load would be amplified of $25 \mathrm{MPa}$. This is beyond the remaining discrepancy between both curves plotted in Fig. 6b.

It is interesting to notice that the oscillations occurring in the load cell response have a repeatable frequency of about $200 \mathrm{~Hz}$ (Fig. 3). Similar oscillations were noticed, with the same frequency, in the standard tensile tests for the highest cross head speed (Fig. 1). This indicates that $200 \mathrm{~Hz}$ is the resonance frequency of the load cell itself or of the whole system, and that is the reason why the vibrations are amplified in the load cell response at this particular frequency.

Therefore, the load cell data is not reliable during the transient zone of the test for deriving the $\sigma_{A V 2}$ stress required by our approach. Between $6 \mathrm{~ms}$ to $15 \mathrm{~ms}$, there is a discrepancy between the far-field stresses (at the load cell location) and the stresses in the measurement area due to the amplified vibrations in the load cell. As $\sigma_{A V 2}$ is not reliable, another way of calibrating $\sigma_{A V 1}$ is needed. Fortunately, the cross section at the top of the measurement area $(y=L)$ remains elastic during the whole duration of the transient effects ( $6 \mathrm{~ms}$ to $15 \mathrm{~ms}$ ). Therefore, it can be used to provide a reliable estimate of the resultant load. Indeed, as $E$ and $v$ were identified during the elastic range, they can be used to derive the following equation:

$$
F\left(t_{l}\right)=\frac{E h}{\left(1-v^{2}\right)} \int_{-w / 2}^{w / 2}\left[\varepsilon_{y y}\left(x, L, t_{l}\right)+v \varepsilon_{x x}\left(x, L, t_{l}\right)\right] d x
$$

This equation provides a new estimate of $\sigma_{A V 2}$, which may be written as:

$$
\sigma_{A V 2 b}\left(t_{l}\right)=\frac{E h L}{\left(1-v^{2}\right) S} \int_{-w / 2}^{w / 2}\left[\varepsilon_{y y}\left(x, L, t_{l}\right)+v \varepsilon_{x x}\left(x, L, t_{l}\right)\right] d x
$$

The evolution of $\sigma_{A V 2 b}$ is plotted in Fig. 9. As expected, there is a drop at the onset of plasticity, but no oscillation is visible. A good agreement with $\sigma_{A V 1}$ is then recovered. Thus, the model given in Eq. (26) is relevant for the actual yield flow in the studied material. This application shows also through Eq. (28) that the requirement of using a load cell can be removed if the elastic properties of the material are known and if full-field deformation measurements are available. Indeed, the strains measured through a given cross section of the material can be used to derive the stresses through this 
cross section, and Eq. (28) can be used to deduce directly the resultant load through this cross section, which replaces the usual load cell.

It is important to point out that the model which has been calibrated in this study takes account of strain localization transient effects induced at the onset of plasticity. This is only possible by coupling a full-field measurement technique and an inverse approach. Transient phenomena are usually not considered by authors who attempt calibration of visco-plastic constitutive models using global response data. By using full-field data, the present results confirm that the transient component of the visco-plastic constitutive response can be modeled so that an aspect of the actual visco-plastic behavior of metals that may be of importance at high strain rates can be adequately characterized.

\section{Conclusions}

In this paper, the identification of elasto-visco-plastic constitutive parameters from full-field deformation measurements was investigated. The shape of the specimen was selected in order to provide non uniform strain rate conditions within the specimen. Using the strain rate heterogeneity, strain fields measured on this single specimen have been shown to provide sufficient information to adequately characterize the elasto-visco-plastic behavior of a typical strain-rate sensitive material using a combination of the VFM with full-field deformation measurements. Specifically, the parameters identified with this approach were compared with the ones obtained using dog-bone specimens. Discrepancies were noticed for the Perzyna's model, due to transient localization effects occurring at the onset of plasticity that were not adequately represented by Perzyna's model. Promising results were obtained using the Yoshida's model, which is a modified version of Perzyna's functional form taking account of Lüders behavior. Eventually, it would be interesting to perform finite element simulations of our experiments, as shown in [20] for instance, for complete validation of the model.

The procedure proposed herein for the quantification of elasto-visco-plastic model parameters, which requires a combination of full-field deformation measurements (with DIC) and an appropriate computational methodology (the VFM) to ensure overall equilibrium and satisfaction of boundary conditions, allowed the investigators to address the issue of transient strain localization at the onset of plasticity in tensile experiments carried out at a high strain rate. Extension to very high strain rates is one of the main prospects for this approach because the transient effects would be augmented. The 
recent drastic improvements in the technology of high-speed cameras will soon provide suitable conditions for these experiments.

\section{References}

1. Lemaître, J. and Chaboche, J.L. Mechanics of Solid Materials. Cambridge University Press, 1990.

2. Kajberg, J., Sundin, K.G., Melin, L.G., Stahle, P. High strain-rate tensile testing and viscoplastic parameter identification using microscopic high-speed photography. International Journal of Plasticity, 20:561-575, 2004.

3. Yoshida, F. A constitutive model of cyclic plasticity. International Journal of Plasticity, 16:359380,2000 .

4. Besnard, G., Hild, F. and Roux, S., "Finite-Element" Displacement Fields Analysis from Digital Images: Application to Portevin-Le Châtelier Bands. Experimental Mechanics, 46(6):789-803, 2006.

5. Schreier, H. and Sutton, M. Systematic errors in digital image correlation due to undermatched subset shape functions. Experimental Mechanics, 42(3):303-310, 2002. Springer.

6. Meuwissen, M.H.H., Oomens, C.W.J., Baaijens, F.P.T., Petterson, R., and Janssen, J.D. Determination of the elasto-plastic properties of aluminium using a mixed numerical-experimental method. Journal of Materials Processing Technology, 75:204-211, 1998.

7. Kajberg, J., Wikman B. Viscoplastic parameter estimation by high strain-rate experiments and inverse modelling - Speckle measurements and high-speed photography. International Journal of Solids and Structures, 44:145-164, 2007.

8. Kajberg, J., Lindkvist G. Characterisation of materials subjected to large strains by inverse modelling based on in-plane displacement fields. International Journal of Solids and Structures, 41:3439-3459, 2004.

9. Grédiac, M. Principe des travaux virtuels et identification. Comptes Rendus de l'Académie des Sciences, 309:1-5, 1989.

10. Grédiac, M., Toussaint, E., and Pierron, F. Special virtual fields for the direct identification of material parameters with the virtual fields method. 1- Principle and definition. International Journal of Solids and Structures, 39(10):2691-2706, 2002. Elsevier.

11. Avril, S., and Pierron, F. General framework for the identification of constitutive parameters from full-field measurements in linear elasticity. International Journal of Solids and Structures, 44:4978-5002, 2007. Elsevier. 
12. Grédiac, M. and Pierron, F. Applying the virtual field method to the identification of elastoplastic constitutive parameters. International Journal of Plasticity, 22:602-627, 2006.

13. Pannier, Y., Avril, S., Rotinat, R., and Pierron, F. Identification of elasto-plastic constitutive parameters from statically undetermined tests using the virtual fields method. Experimental Mechanics, 46(6), 735-755, 2006.

14. Pannier, Y., Identification of elasto-plastic parameters from statically-undetermined tests: experimental application and validation of the virtual fields method (in French). PhD Thesis, Ecole nationale Supérieure d'Arts et Métiers, CER de Châlons en Champagne, France, 2006.

15. Avril, S., Pannier, Y., Pierron, F., and Rotinat, R. Stress reconstruction and constitutive parameter identification in plane-stress elasto-plastic problems using surface measurements of deformation fields. Experimental Mechanics, in revision, 2007.

16. Sun, H.B., Yoshida, F., Ma, X., Kamei, T., and Ohmori, M. Finite element simulation on the propagation of Lüders band and effect of stress concentration. Materials Letters, 57:3206-3210, 2003.

17. Graff, S., Forest, S., Strudel, J.-L., Prioul, C., Pilvin, P. and Béchade, J.-L. Strain localization phenomena associated with static and dynamic strain ageing in notched specimens: experiments and finite element simulations. Materials Science and Engineering A, 387-389:181-185, 2004.

18. VIC2D, Correlated Solutions Incorporated, 952 Sunset Blvd, West Columbia, SC, www.correlatedsolutions.com.

19. Sutton, M.A., Deng, X., Liu, J., and Yang, L. Determination of elastic plastic stresses and strains from measured surface strain data. Experimental Mechanics, 36(2):99-112, 1996.

20. Kyriakides, S., Miller, J.E. On the propagation of Lüders band in steel strips. Journal of Applied Mechanics, 67(4):645-654, 2000. 
List of tables:

1. Reference values of the constitutive parameters at different strain rates.

2. Elastic parameters and constitutive parameters of Perzyna's model identified with the VFM....... 24

3. Elastic parameters and constitutive parameters of modified Perzyna's model identified with the VFM. 
Tab. 1. Reference values of the constitutive parameters at different strain rates.

\begin{tabular}{|l|c|c|c|c|c|c|}
\hline Loading rate & $0.01 \mathrm{~mm} / \mathrm{s}$ & $2.54 \mathrm{~mm} / \mathrm{s}$ & $6.35 \mathrm{~mm} / \mathrm{s}$ & $15 \mathrm{~mm} / \mathrm{s}$ & $25.4 \mathrm{~mm} / \mathrm{s}$ & $63.5 \mathrm{~mm} / \mathrm{s}$ \\
\hline Strain rate & $2 \times 10^{-4} \mathrm{~s}^{-1}$ & $4.2 \times 10^{-2} \mathrm{~s}^{-1}$ & $0.105 \mathrm{~s}^{-1}$ & $0.25 \mathrm{~s}^{-1}$ & $0.42 \mathrm{~s}^{-1}$ & $1.05 \mathrm{~s}^{-1}$ \\
\hline$Y_{0}$ & $190 \mathrm{MPa}$ & $249 \mathrm{MPa}$ & $255 \mathrm{MPa}$ & $267 \mathrm{MPa}$ & $279 \mathrm{MPa}$ & $296 \mathrm{MPa}$ \\
\hline$E_{t}$ & $2400 \mathrm{MPa}$ & $970 \mathrm{MPa}$ & $960 \mathrm{MPa}$ & $830 \mathrm{MPa}$ & $770 \mathrm{MPa}$ & $700 \mathrm{MPa}$ \\
\hline
\end{tabular}


Tab. 2. Elastic parameters and constitutive parameters of Perzyna's model identified with the VFM.

\begin{tabular}{|l|c|c|c|c|c|c|}
\hline & $E(\mathrm{GPa})$ & $v$ & $\sigma_{0}(\mathrm{MPa})$ & $E_{t}(\mathrm{GPa})$ & $\gamma\left(\mathrm{s}^{-1}\right)$ & $n$ \\
\hline Spec. 1, Cam. 1 & 203 & 0.27 & 156 & 0.40 & 0.16 & 19 \\
\hline Spec. 1, Cam. 2 & 203 & 0.32 & 156 & 0.80 & 0.48 & 10.1 \\
\hline Spec. 2, Cam. 1 & 198 & 0.30 & 145 & 0.38 & 0.15 & 10.4 \\
\hline Spec. 2, Cam. 2 & 198 & 0.32 & 152 & 0.14 & 0.22 & 13.6 \\
\hline Reference & $199 \pm 5$ & $0.28 \pm 0.03$ & 140 & $\mathrm{x}$ & 0.5 & 8 \\
\hline
\end{tabular}


Tab. 3. Elastic parameters and constitutive parameters of modified Perzyna's model identified with the VFM.

\begin{tabular}{|l|c|c|c|c|c|c|c|c|}
\hline & $\begin{array}{c}E \\
(\mathrm{GPa})\end{array}$ & $v$ & $\begin{array}{c}\sigma_{0} \\
(\mathrm{MPa})\end{array}$ & $\begin{array}{c}E_{t} \\
(\mathrm{GPa})\end{array}$ & $n$ & $\begin{array}{c}\gamma_{0} \\
\left(\mathrm{~s}^{-1}\right)\end{array}$ & $\begin{array}{c}\gamma_{\infty} \\
\left(\mathrm{s}^{-1}\right)\end{array}$ & $\lambda$ \\
\hline Spec. 1, Cam. 1 & 203 & 0.27 & 134 & 1.6 & 5.4 & 0.007 & 0.2 & 26 \\
\hline Spec. 1, Cam. 2 & 203 & 0.32 & 122 & 1.2 & 5.6 & 0.004 & 0.1 & 15 \\
\hline Spec. 2, Cam. 1 & 198 & 0.30 & 140 & 1 & 10.2 & 0.007 & 0.17 & 29 \\
\hline Spec. 2, Cam. 2 & 198 & 0.32 & 122 & 1.2 & 8.8 & 0.005 & 0.23 & 28 \\
\hline Reference & $\begin{array}{c}199 \\
\pm 5\end{array}$ & $\begin{array}{c}0.28 \\
\pm 0.03\end{array}$ & 140 & $\mathrm{x}$ & 8 & $\mathrm{x}$ & 0.5 & $\mathrm{x}$ \\
\end{tabular}


List of figures:

1. Stress/strain curves obtained at different strain rates in the standard uniaxial tensile tests on the dogbone specimens.

2. Sketch of the non-standard specimens with the measurement area meshed using triangles for reconstruction of displacement fields.

3. Measured resultant loads $F(t)$ during both experiments.

4. Maps of strain component $\varepsilon_{y y}$ during experiment (Specimen 2, Camera 1). 30

5. Fields of strain rate during experiment (Specimen 2, Camera 1).

6. Curves of $\sigma_{A V 1}$ and $\sigma_{A V 2}$ deduced for specimen 2, (a) Raw Perzyna's model, (b) Modified Perzyna's model with Lüders behaviour..

7. Comparison of the viscoplastic models identified using the standard approach (dogbone specimen) or using the VFM (with the double notched specimen) with the two tested models (Perzyna and modified Perzyna)

8. Field of vertical acceleration (Specimen 2, Camera 1)

9. Curves of $\sigma_{A V 1}$ (from whole measurement area) and $\sigma_{A V 2 b}$ (from elastic strains only) plotted up to $14 \mathrm{~ms}$ deduced for specimen 2 . 


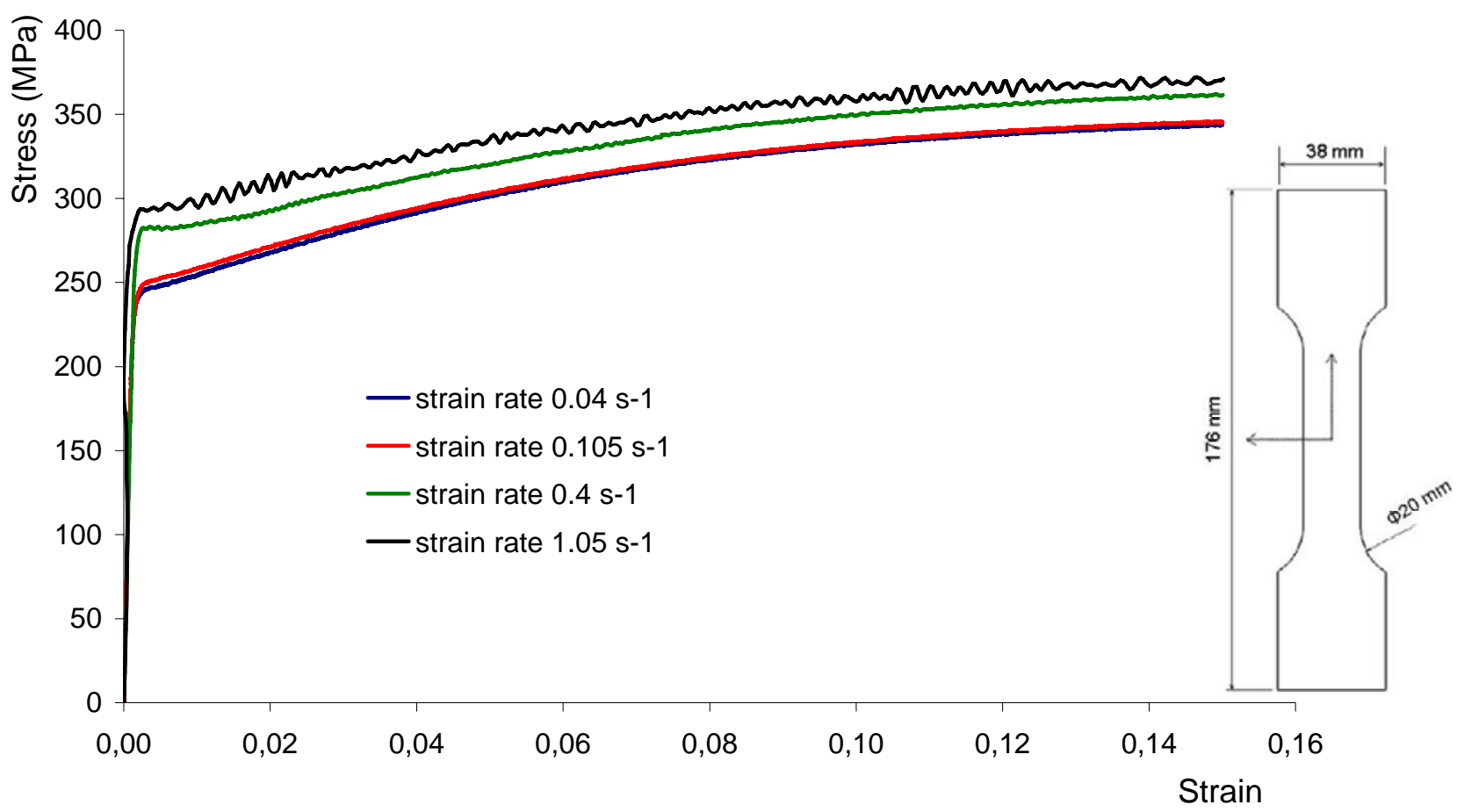

Fig. 1. Stress/strain curves obtained at different strain rates in the standard uniaxial tensile tests on the dogbone specimens. 


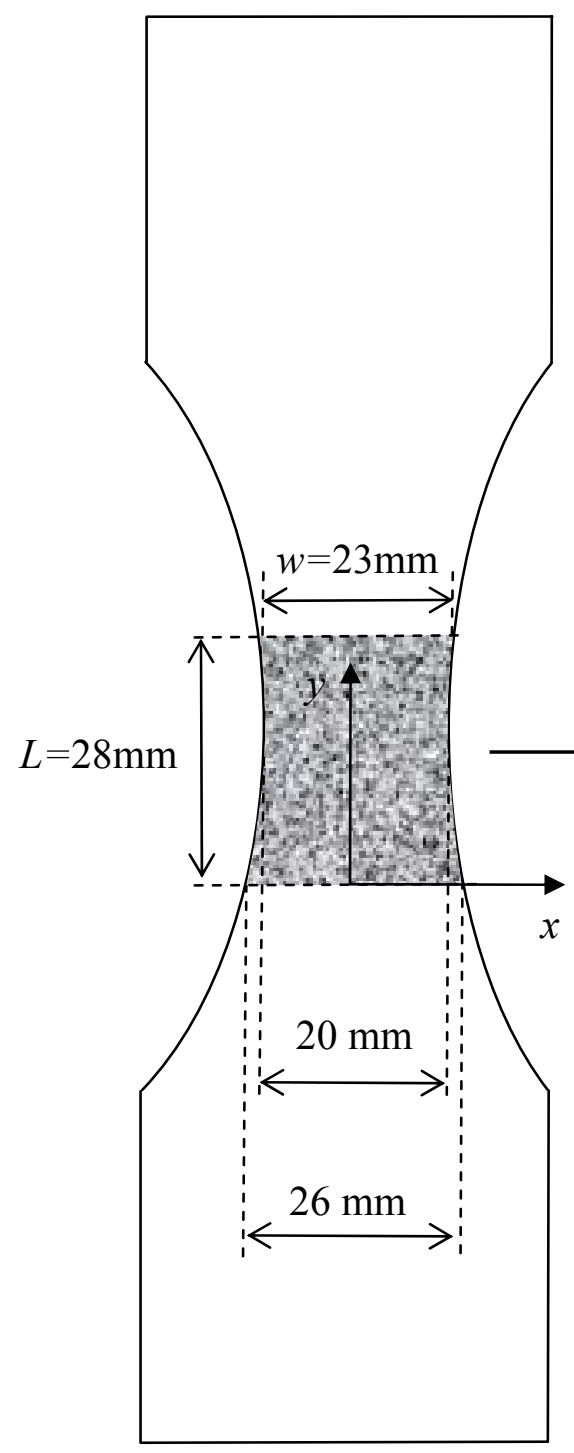

Meshing of the area of measurements $S$ into triangles with a mesh size of $4 \mathrm{~mm}$ in average

Fig. 2. Sketch of the non-standard specimens with the measurement area meshed using triangles for reconstruction of displacement fields 


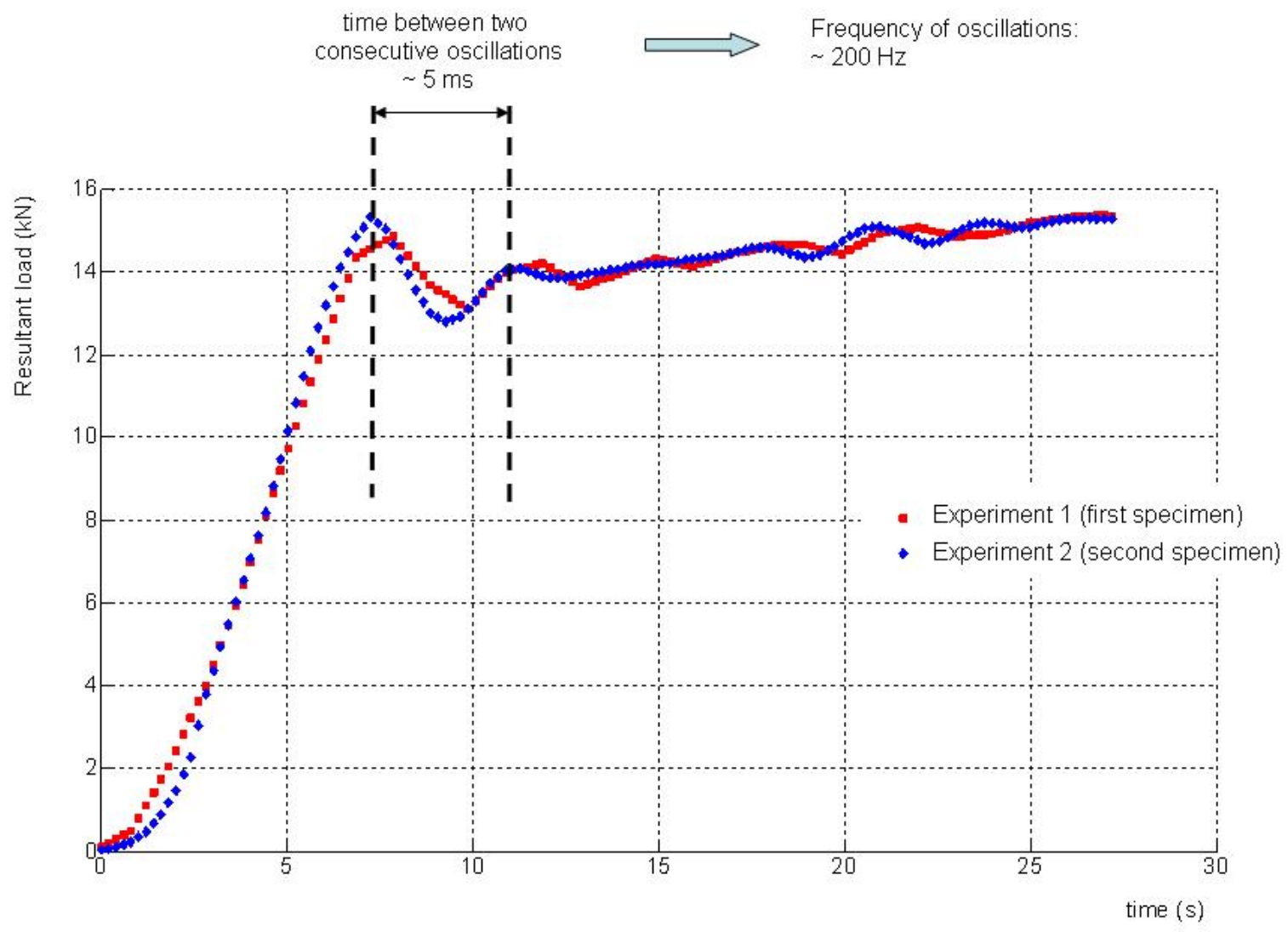

Fig. 3: Measured resultant load $F(t)$ during both experiments. 

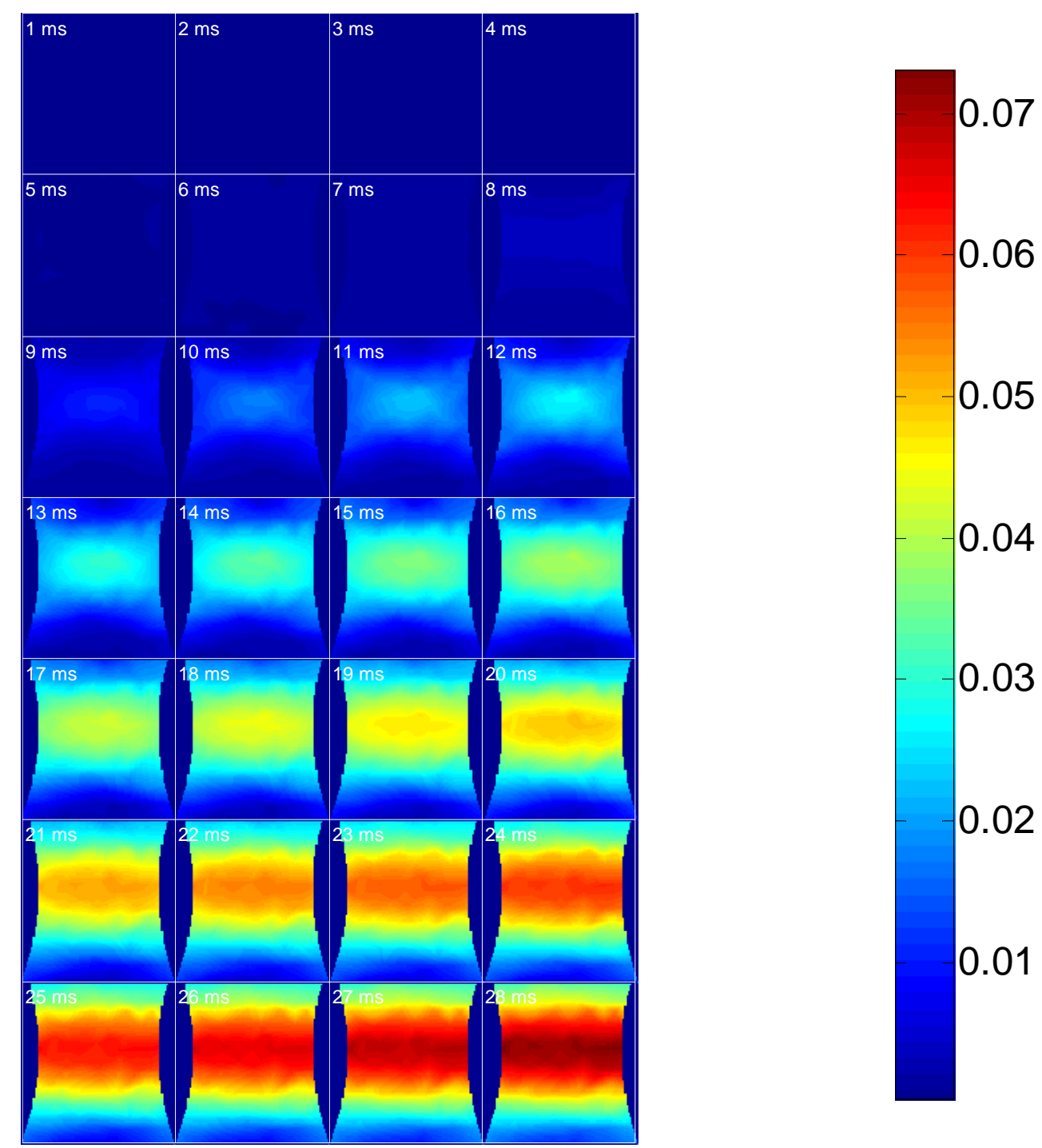

Fig. 4: Maps of strain component $\varepsilon_{y y}$ during experiment (Specimen 2, Camera 1). 


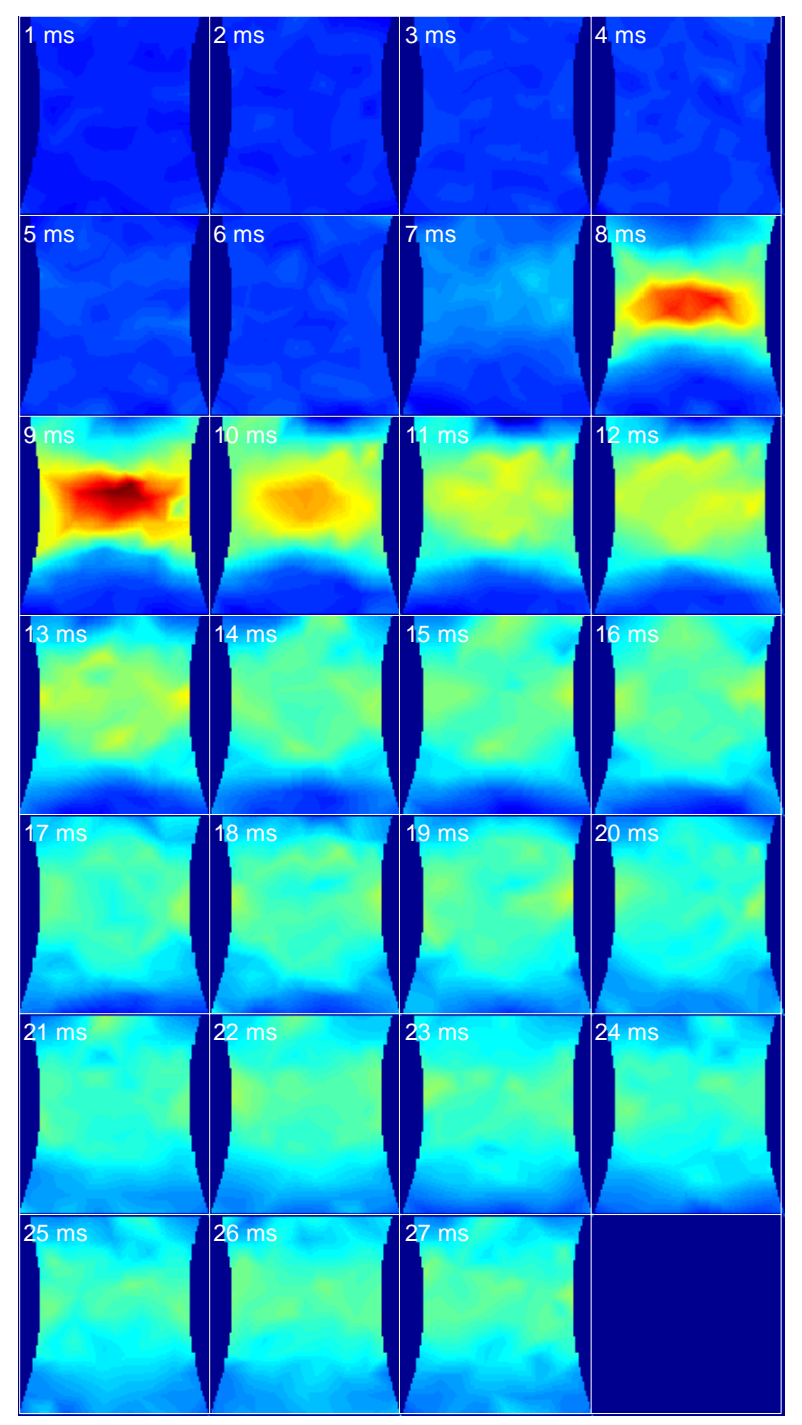

\section{strain rate}

$\left(\mathrm{s}^{-1}\right)$

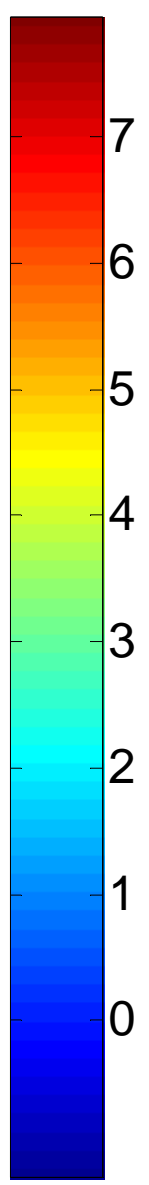

Fig. 5: Fields of strain rate during experiment (Specimen 2, Camera 1). 


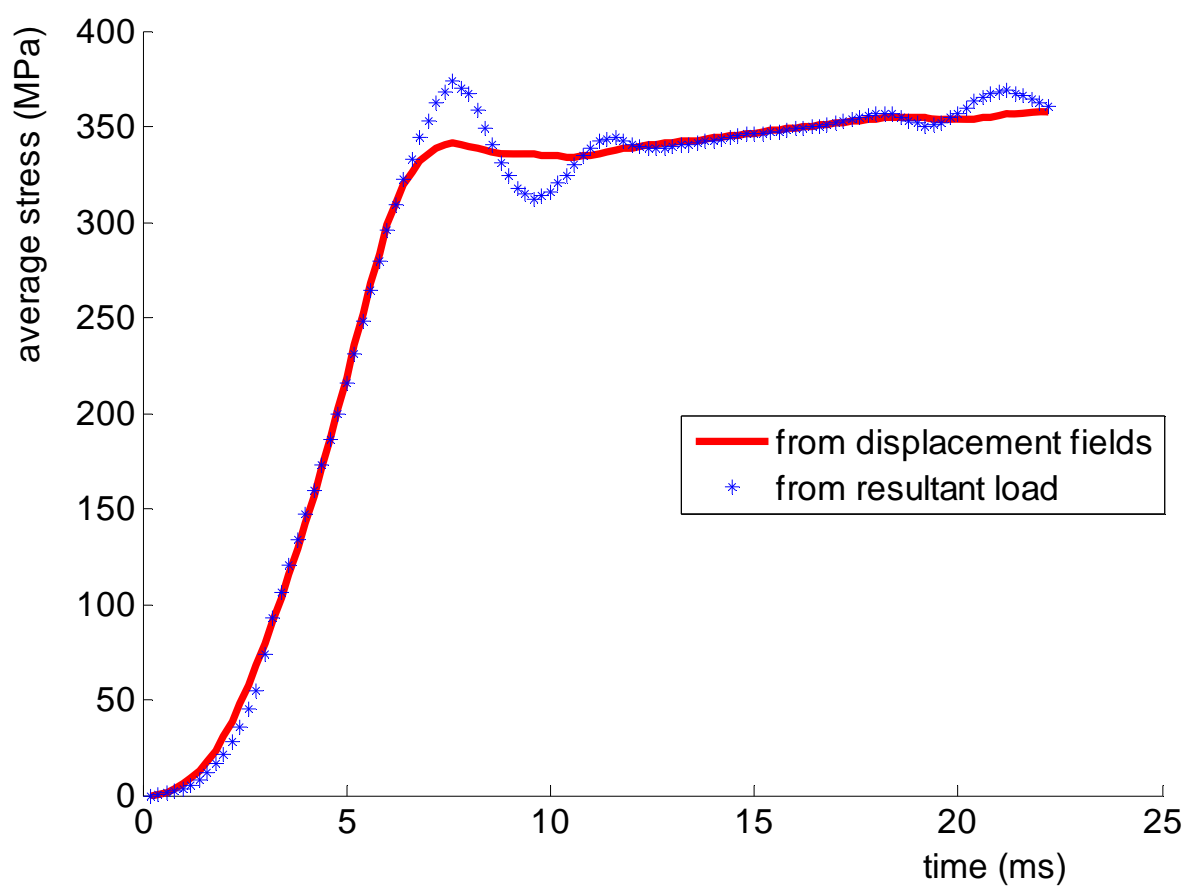

(a) Raw Perzyna's model

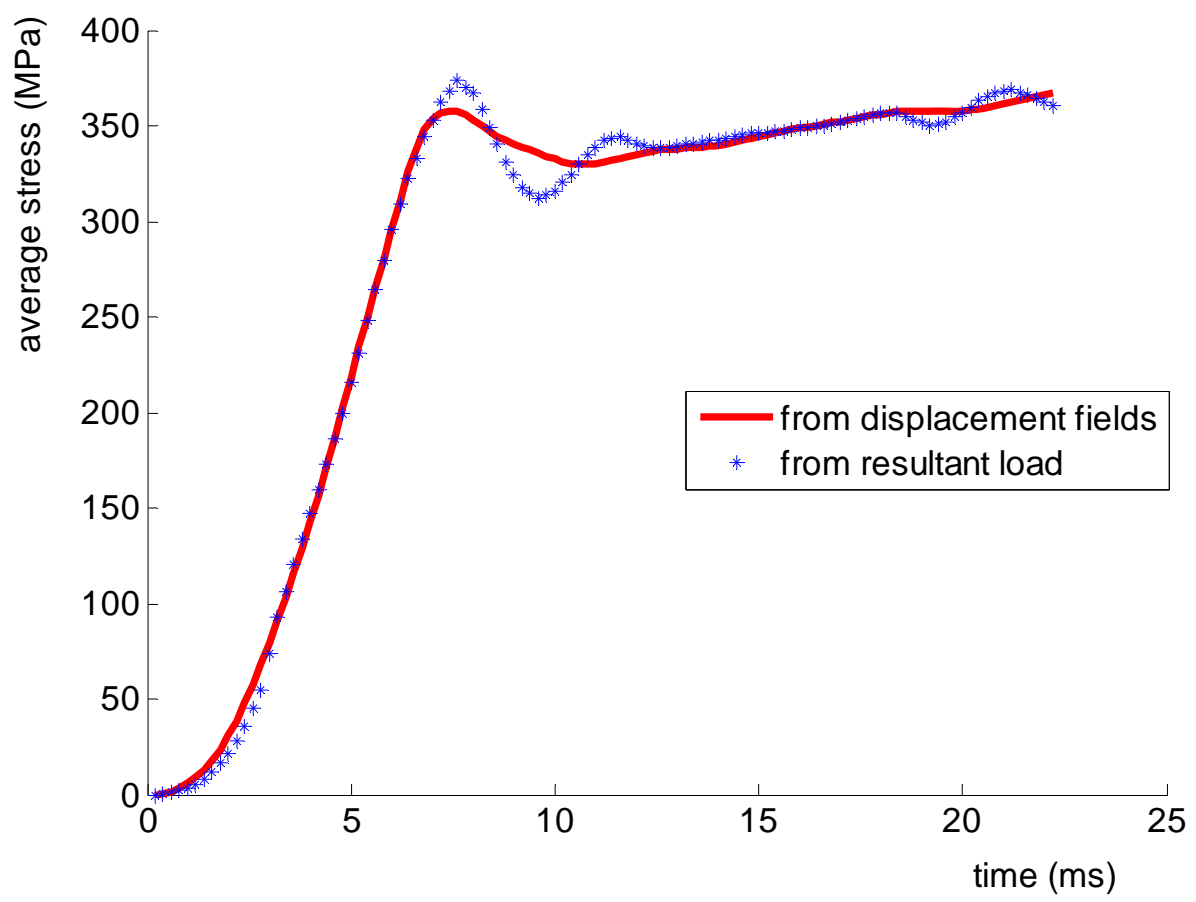

(b) Modified Perzyna's model with Lüders behaviour

Fig. 6: Curves of $\sigma_{A V 1}$ (from displacement fields) and $\sigma_{A V 2}$ (from resultant load) deduced for specimen 2. 
- model identified using the standard approach

— model identified using the VFM with modified Perzyna

- - model identified using the VFM with Perzyna

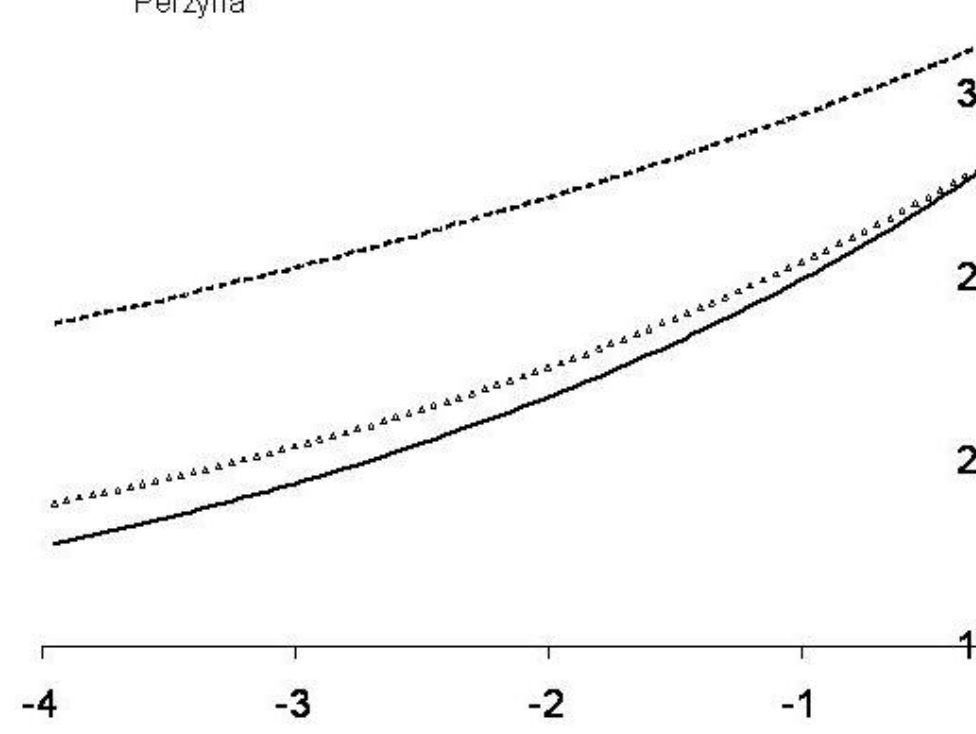

Fig. 7: Comparison of the viscoplastic models identified using the standard approach (dogbone specimen) or using the VFM (with the double notched specimen) with the two tested models (Perzyna and modified Perzyna). 


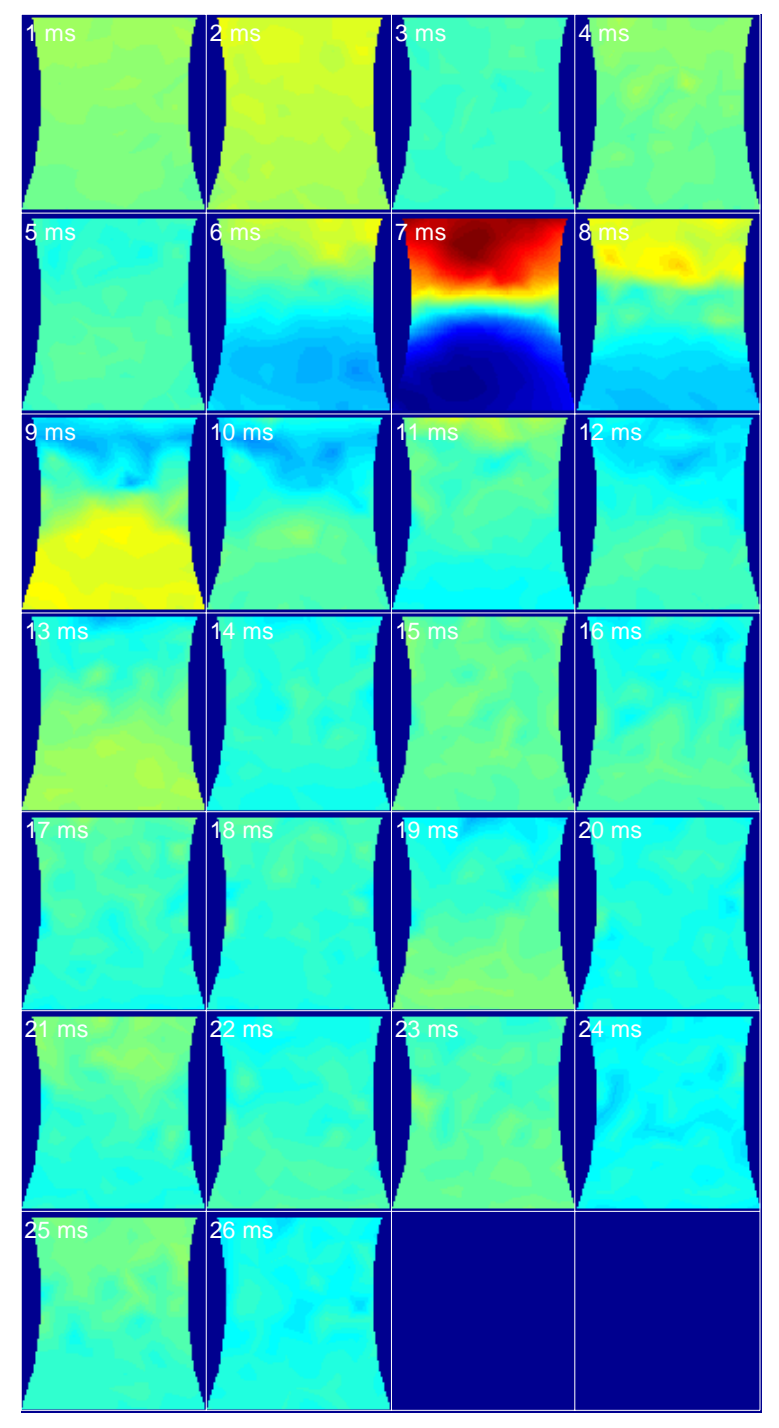

acceleration
in $\mathrm{g}$

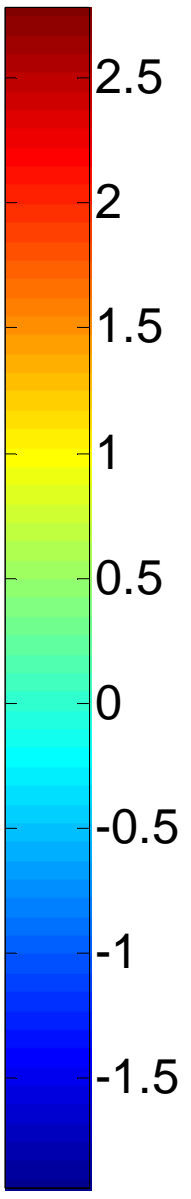

Fig. 8: Field of vertical acceleration (Specimen 2, camera 1). 


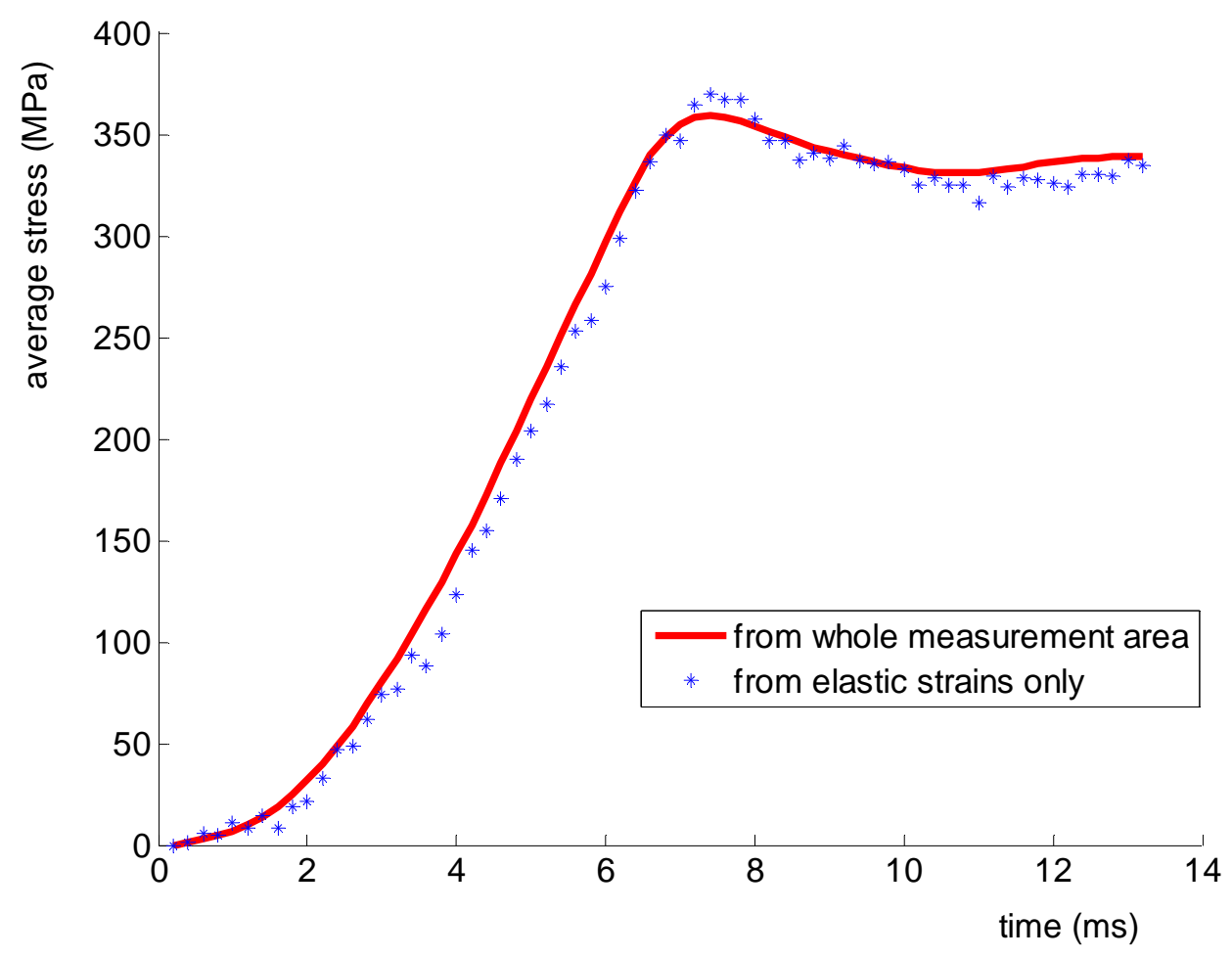

Fig. 9: Curves of $\sigma_{A V 1}$ (from whole measurement area) and $\sigma_{A V 2 b}$ (from elastic strains only) plotted up to $14 \mathrm{~ms}$ deduced for specimen 2 . 